Journal of Accident and Emergency Medicine 1995 12, 286-287

\title{
Do patients presenting to accident and emergency departments with the sensation of a foreign body in the eye (gritty eye) have significant ocular disease?
}

\author{
D.G.R. JAYAMANNE \\ Department of Ophthalmology, Newcastle General Hospital, Newcastle Upon Tyne, UK
}

\section{SUMMARY}

A total of 1155 consecutive patients selected on symptomatology alone who presented to an ophthalmic accident and emergency (A\&E) department over a period of 3 months from a total patient population of $\mathbf{3 5 2 2}$ were assessed. The study showed that about $96 \%$ of the patients reporting a sensation of a foreign body in their eye had no serious ocular disease. Most patients had a superficial mechanical injury or minor microbial infection. Steroid drugs were prescribed to only $2.1 \%$ of the patients. Topical steroid treatment was contraindicated in $\mathbf{0 . 3 5 \%}$ of the patients. A low rate of morbidity was noted, suggesting that these patients could have been treated adequately in a general A\&E department rather than an ophthalmic department.

Key words: eyes, foreign bodies, steroid treatment

\section{INTRODUCTION}

The sensation of a foreign body in the eye (gritty eye) is a common ocular symptom. ${ }^{1}$ Although most of these patients do not have significant ocular disease, it is important to carry out a careful clinical examination. Significant ocular disorders such as marginal keratitis and episcleritis may present with gritty, irritable eyes. ${ }^{2}$ The aim of this study was to identify the causes of the sensation of a foreign body in the eye in a group of 1155 new patients presenting to an ophthalmic A\&E department. The study also reports the indications and incidence of topical steroid use in this group of patients.

Medical practitioners are advised never to prescribe topical ophthalmic steroids without first seeking the opinion of an ophthalmologist. ${ }^{2}$ Disorders for which steroid treatment may be beneficial include marginal keratitis, blepharitis, allergic conjunctivitis, episcleritis, uveitis and viral conjunctivitis. Disorders for which steroid treatment is contraindicated include bacterial keratitis and herpes simplex keratitis. Non-ophthalmic trained medical practitioners show a high level of anxiety about the management of ophthalmic disease. ${ }^{3}$ Inappropriate use of topical ophthalmic steroid preparations can potentially result in significant visual handicap from steroid-induced glaucoma or cataracts. ${ }^{2}$ Accurate diagnosis of ocular disease is particularly important before treatment with topical steroids. This depends on examination using a slit lamp biomicroscope and other specialist ophthalmic techniques. ${ }^{3}$

\section{PATIENTS AND METHODS}

During the 3 month study period, 3522 patients presented to the ophthalmic A\&E department at the North Riding Infirmary, Cleveland, UK for the first time; the patients were either self-referred or referred by their general practitioners (GPs). About 12000 new patients are seen in the department every year.

Selection criteria for inclusion in this study were based on symptomatology alone. Patients in whom a sensation of a foreign body in the eye was identified to be the most significant symptom were included in the study. A total of 1155 new patients satisfied these criteria. The clinical records of these patients were reviewed retrospectively. All ocular examinations were performed by a junior ophthalmologist. These included visual acuity measurement, bio-microscopic examination, eversion of the eyelids and fluorescein staining. Direct and consensual pupil reactions were also noted on all patients. Orbital radiographs were performed on all patients with a 
D.G.R. Jayamanne history of high velocity injury (e.g. hammering or drilling metal on metal).

\section{RESULTS}

During the 3 months of the study about $35 \%$ of new patients presenting to the A\&E department stated that the sensation of a foreign body in the eye was the most significant ocular symptom that affected them at the time of presentation. A total of $13 \%$ of the patients reported solely such a sensation in the eye, with no other associated symptoms. The sensation of a foreign body was associated with lacrimation in almost $50 \%$ of patients. Three per cent of patients with a corneal foreign body required an orbital radiograph to exclude an intra-ocular foreign body.

By far the most common cause of the sensation of a foreign body in this group of $\mathbf{1 1 5 5}$ patients was a superficial non-penetrating corneal foreign body. About $75 \%$ of these patients had a clear history of trauma. The results of this study are summarized in Table 1.

\section{DISCUSSION}

There are limitations to this type of study. Over the study period the prevalence data represent only disease in patients who sought hospital treatment at the time and exclude all those patients receiving

Table 1. Incidence of each cause of the sensation of foreign body in the eye as a percentage of the total number of patients in the study $(n=1155)$

\begin{tabular}{lc}
\hline Diagnosis & Incidence \\
\hline Corneal foreign body & 67.8 \\
Corneal abrasion & 13.6 \\
Bacterial/viral conjunctivitis & 9.1 \\
None found & 2.9 \\
Allergic conjunctivitis & 1.6 \\
Marginal keratitis & 1.4 \\
Tarsal cyst & 0.8 \\
Blepharitis & 0.8 \\
Dry eye syndrome & 0.6 \\
Episcleritis & 0.4 \\
Herpes simplex keratitis & 0.3 \\
Trichiasis/entropion & 0.3 \\
Anterior uveitis & 0.2 \\
Contact lens keratitis & 0.2 \\
\hline
\end{tabular}

treatment from GPs. Despite the limitations, however, this study provides information on the incidence of acute disorders in the community.

By far the largest percentage of patients with the sensation of a foreign body in the eye had mechanical injuries to the eye such as foreign bodies, glancing blows and other superficial trauma causing corneal abrasions. Microbial conjunctivitis and seasonal allergic conjunctivitis were the most common non-traumatic disorders in this study. The incidence of herpes simplex keratitis presenting as the sensation of a foreign body in the eye was low. Fluorescein staining revealed all herpetic, marginal and traumatic ulceration of the cornea, and should therefore be carried out routinely in all patients with such a sensation in the eye.

Most ophthalmologists are concerned about the inappropriate use of steroid treatment by nonophthalmic medical practitioners and feel that steroid preparations should not be used without specialist advice. ${ }^{4}$ In this group of patients, which included about $35 \%$ of all new patients attending the ophthalmic A\&E department, only $2.1 \%$ of patients required a steroid preparation as a part of their treatment. The ocular disorders for which steroid treatment was essential included patients with uveitis, marginal keratitis and episcleritis. In this study only four patients $(0.35 \%)$ had disorders strongly contraindicating the use of topical steroid treatment, i.e. herpes simplex keratitis.

A low rate of morbidity noted in most patients provides strong evidence that ophthalmic emergency services can be provided adequately by general departments, but close liaison with the ophthalmology departments should be maintained so that rapid access is available for patients with complicated ophthalmic disease.

\section{REFERENCES}

1. Thiry D. (1979) Ocular symptomatology in general practice. Revue Medicale de Liege 34, 803-810.

2. Claoue C.M.P. \& Stevenson K.E. (1986) incidence of inappropriate treatment of herpes simplex keratitis with topical steroids. British Medical Joumal 292, 1450-1451.

3. Wilson A. (1987) The red eye: a general practice survey. Journal of the Royal College of General Practitioners 37, 62-64.

4. St Clair Roberts D. (1986) Steroids, the eye and general practitioner. British Medical Journal 292, 1414-1415. 